\title{
Educación para la salud en preescolares de guarderías
}

\author{
Elsa Carolina Rojas-Ortiz, Vidal E. Álvarez-Rodríguez, Hilda Gabriela León-Suazo, \\ Arturo Baños-Sánchez,Patricia Trejo-Morales Y Demetrio Arturo Bernal-Alcántara* \\ Delegación Regional Zona Poniente, Instituto de Seguridad y Servicios Sociales de los Trabajadores del Estado, Ciudad de México, México
}

\section{Resumen}

Antecedentes: La promoción de la salud se realiza en servicios preventivos de unidades médicas, en programas educativos y libros de texto desde la perspectiva general de la población. Objetivo: Determinar características basales de educación en salud a preescolares, sus padres y maestros, mediante médicos especialistas. Método: Estudio transversal de un programa educativo para preescolares en estancias infantiles. Se evaluaron habilidades para la vida y competencias para la salud. Resultados: Los preescolares desarrollaron resiliencia (84.12\%), empatía (92.23\%), comunicación asertiva (93.5\%), relaciones interpersonales (91.0\%), toma correcta de decisiones correctas (92.56\%), solución de problemas (81.05\%), pensamiento creativo (98\%), pensamiento crítico (86.08\%), manejo de emociones (80.76\%), activación física (97.94\%), autoconocimiento (98.96\%), hábitos higiénicos (94.90\%) y corresponsabilidad (86.25\%); al 95.59\% les agradó el taller y el 95.75\% eligió la opción correcta en los problemas descritos. El 63.04\% de los padres generaron cambios en sus hábitos y en los de sus hijos. Conclusión: El programa educativo implementado puede favorecer el empoderamiento en salud desde la infancia.

PALABRAS CLAVE: Preescolares. Promoción de la salud. Resiliencia.

\begin{abstract}
Background: The promotion of health is carried out in preventive services of medical units, in educational programs and textbooks from the general perspective of the population. Objective: To determine baseline characteristics of health education for pre-school children, their parents and teachers, by means of specialist doctors. Method: Cross-sectional study of an educational program for preschool children. Life skills and health competencies were evaluated. Results: The preschoolers developed resilience (84.12\%), empathy (92.23\%), assertive communication (93.5\%), interpersonal relationships (91.0\%), correct decision making (92.56\%), problem solving (81.05\%), creative thinking (98\%), critical thinking (86.08\%), emotion management (80.76\%), physical activation (97.94\%), self-knowledge (98.96\%), hygienic habits (94.90\%) and co-responsibility (86.25\%); 95.59\% liked the workshop and $95.75 \%$ chose the correct option in the problems described. $63.04 \%$ of parents generated changes in their habits and those of their children. Conclusion: The educational program implemented can promote empowerment in health from childhood.
\end{abstract}

KEY WORDS: Preschoolers. Health promotion. Resilience.

Correspondencia:

*Demetrio Arturo Bernal Alcántara

E-mail: demetrio.bernal@issste.gob.mx
Fecha de recepción: 06-03-2019

Fecha de aceptación: 19-03-2019

DOI: 10.24875/GMM.19005139
Gac Med Mex. 2019;155(Suppl 1):S38-S44 Disponible en PubMed www.gacetamedicademexico.com 


\section{Introducción}

La familia, la escuela y los sistemas de salud fungen como factores protectores si el individuo encuentra en ellos seguridad, apoyo e información que le permita tomar decisiones que beneficien su calidad de vida $^{1,2}$; sin embargo, esto no está ocurriendo en la sociedad contemporánea, la cual se considera de riesgo ${ }^{3}$.

México está dentro de los primeros cinco lugares en sobrepeso y obesidad infantil y ocupa el segundo lugar en la de adultos ${ }^{4}$, lo cual representa un factor de riesgo, ya que reduce la esperanza y calidad de vida al producir enfermedades como diabetes, hipertensión arterial, dislipidemias y problemas psicológi$\cos ^{5}$. A nivel mundial, la anorexia y la bulimia se encuentran entre los trastornos alimentarios que van en aumento ${ }^{6}$.

Actualmente, México ocupa el primer lugar de embarazos en adolescentes según la Organización para la Cooperación y el Desarrollo Económico y de acuerdo con la Encuesta Nacional de la Dinámica Demográfica 2014, una de cada tres adolescentes del grupo de 15 a 19 años ya inició su vida sexual y de ese total, más del $40 \%$ no utilizó algún método anticonceptivo en su primera relación sexual, lo que se considera una conducta de riesgo que puede afectar su proyecto de $v^{\text {vida }}{ }^{7,8}$. Asimismo, México ocupa el primer lugar en abuso sexual y violencia física infantil y a pesar de las guías de actuación y de algunos programas de prevención, hay todavía mucho trabajo por realizar9,10.

El manejo de emociones es uno de los aspectos básicos que garantizan la salud, sin embargo, algunos niños y adolescentes tienen dificultades para lograrlo y generan conductas autolesivas,depresión, ansiedad, estrés 0 adicciones ${ }^{11,12}$.

La depresión es un trastorno mental que afecta el desempeño familiar, escolar, laboral y social de la persona que la padece y su aparición en edades tempranas se considera un factor de riesgo de comorbilidad en la edad adulta ${ }^{13-15}$.

Actualmente, las lesiones autoinfligidas se encuentran dentro de las tres primeras causas de mortalidad en el grupo de 15 a 24 años, lo que representa más de la mitad de las defunciones totales de este grupo ${ }^{16}$.

La dinámica familiar y escolar se ve afectada por el consumo de sustancias nocivas para la salud como tabaco, alcohol o drogas (marihuana, inhalables, tranquilizantes y cocaína) que se presenta desde edades tempranas ${ }^{17-19}$ y el acoso escolar o bullying que afecta la salud física y emocional de los agresores, las víctimas y los espectadores ${ }^{20}$. Otra variable que afecta esta dinámica es el manejo inadecuado de niños y jóvenes con trastorno por déficit de atención e hiperactividad, ya que representa uno de los motivos de consulta más frecuentes en la especialidad de psiquiatría infantil ${ }^{21}$ y se estima que tiene una prevalencia aproximada del $5 \%{ }^{22}$. Frente a este panorama epidemiológico, los responsables de la salud requieren impulsar programas preventivos que permitan el desarrollo de conductas y hábitos saludables desde la infancia para evitar conductas de riesgo a lo largo de la vida. Con el objetivo de contribuir al control y prevención de las enfermedades crónicas y disminuir algunos de los problemas sociales, se creó el Programa ISSSTE en tu Escuela (del Instituto de Seguridad y Servicios Sociales de los Trabajadores del Estado), el cual tiene como eje rector la resiliencia. La resiliencia es la capacidad de los seres humanos de afrontar las adversidades superándolas, resurgiendo, adaptándose y reconstruyéndose para fomentar un desarrollo psicológico y social exitoso ${ }^{23,24}$. Al ser un proceso dinámico,esta se puede enseñar en cualquier momento del ciclo vital para lograr un equilibrio entre la personalidad del individuo, los factores de riesgo y los factores de protección ${ }^{25,26}$. Por todo lo anterior, el propósito de esta investigación fue determinar las características basales del Modelo Proactivo de Salud mediante la capacitación de niñas, niños, padres de familia y docentes para generar competencias para la salud y habilidades para la vida y así fortalecer el binomio salud-educación.

\section{Método}

Estudio transversal con muestreo no probabilístico intencional realizado en las Estancias de Bienestar y Desarrollo Infantil (EBDI) de la Delegación Regional Poniente del ISSSTE durante el Ciclo Escolar 20162017. Se impartieron talleres lúdicos-vivenciales a padres de familia, docentes, alumnas y alumnos de Preescolar 2 y 3 . Todos los participantes firmaron la Carta de Consentimiento Informado; además, los padres de familia dieron su autorización por escrito para que sus hijos e hijas participaran en los talleres. El protocolo de investigación fue aprobado por el Comité de Ética e Investigación. La asistencia de las niñas y niños que fueron capacitados en cada uno de los ejes temáticos varió en función del día en que se impartió el taller (rango de 205 a 239). Del total de la muestra, el $52.77 \%$ eran niñas y el $47.23 \%$ fueron niños. 
Tabla 1. Ejes temáticos del Programa ISSSTE en tu Escuela, temas y actividades

\begin{tabular}{|c|c|c|c|c|}
\hline \multicolumn{2}{|c|}{ Eje temático } & \multirow{2}{*}{$\begin{array}{l}\text { Objetivo } \\
\text { Proporcionar herramientas y } \\
\text { habilidades para la vida que } \\
\text { promuevan una conducta resiliente } \\
\text { para enfrentar los retos del futuro }\end{array}$} & \multirow{2}{*}{$\begin{array}{l}\text { Temas } \\
\text { Resiliencia, autoestima, clima } \\
\text { laboral, toma de decisiones, } \\
\text { inclusión y pensamiento creativo }\end{array}$} & \multirow{2}{*}{$\begin{array}{l}\text { Actividades } \\
\text { Club, botiquín y casita resiliente, } \\
\text { arte y resiliencia y el color de mi } \\
\text { adversidad }\end{array}$} \\
\hline I & Resiliencia & & & \\
\hline$\|$ & $\begin{array}{l}\text { Nutrición } \\
\text { saludable desde } \\
\text { la infancia }\end{array}$ & $\begin{array}{l}\text { Proporcionar los conocimientos } \\
\text { y habilidades necesarias para } \\
\text { la toma correcta de decisiones } \\
\text { relacionas con la alimentación y } \\
\text { actividad física }\end{array}$ & $\begin{array}{l}\text { Sobrepeso y obesidad, plato del } \\
\text { buen comer, jarra del bien beber, } \\
\text { lunch saludable, comida no sana, } \\
\text { anorexia y bulimia y actividad física }\end{array}$ & $\begin{array}{l}\text { Cuento de Marian, Jeopardy, } \\
\text { triángulo de la salud, bicicleta } \\
\text { resiliente, preparación de menús } \\
\text { saludables y ejercicios físicos }\end{array}$ \\
\hline III & $\begin{array}{l}\text { Salud mental y } \\
\text { emocional }\end{array}$ & $\begin{array}{l}\text { Adquisición de habilidades para el } \\
\text { control y manejo de emociones que } \\
\text { permitan enfrentar las adversidades } \\
\text { y prevenir enfermedades }\end{array}$ & $\begin{array}{l}\text { Identificación, control y manejo de } \\
\text { emociones, depresión, suicidio, } \\
\text { autoestima, trastorno por déficit de } \\
\text { atención y bullying }\end{array}$ & $\begin{array}{l}\text { Semáforo, panel de emociones, } \\
\text { monstruo come miedos, ardilla } \\
\text { miedosa, expresión corporal y } \\
\text { risoterapia }\end{array}$ \\
\hline IV & $\begin{array}{l}\text { Conociendo } \\
\text { y cuidado mi } \\
\text { cuerpo }\end{array}$ & $\begin{array}{l}\text { Enseñar las competencias que } \\
\text { favorezcan la toma correcta de } \\
\text { decisiones para una adecuada vida } \\
\text { sexual, libre de riesgos y que no } \\
\text { afecten su proyecto de vida }\end{array}$ & $\begin{array}{l}\text { Ciclo de vida, hábitos higiénicos, } \\
\text { embarazo y prevención de abuso } \\
\text { sexual }\end{array}$ & $\begin{array}{l}\text { Identificar las partes del } \\
\text { cuerpo, cuenta cuentos, hábitos } \\
\text { higiénicos, la mano de Kiko y } \\
\text { teatro guiñol }\end{array}$ \\
\hline V & $\begin{array}{l}\text { Habilidades } \\
\text { sociales }\end{array}$ & $\begin{array}{l}\text { Aprender habilidades y } \\
\text { competencias para adoptar un } \\
\text { estilo de vida sano y mejorar la } \\
\text { comunicación en la familia }\end{array}$ & $\begin{array}{l}\text { Concepto de familia y tipos, } \\
\text { comunicación, cinco lenguajes } \\
\text { del amor, valores, normas y } \\
\text { límites, inclusión, prevención de } \\
\text { accidentes y buenos modales }\end{array}$ & $\begin{array}{l}\text { Profesiones y oficios, valores } \\
\text { universales, prevención } \\
\text { de accidentes, botiquín de } \\
\text { emergencia, buenos modales, } \\
\text { estrategias de comunicación y } \\
\text { manejo de límites }\end{array}$ \\
\hline
\end{tabular}

Los responsables de cada eje temático aplicaron una encuesta a las niñas y niños en la que, de manera individual, expresaron su grado de satisfacción y resolvieron un problema que permitió medir su capacidad para aplicar los conocimientos adquiridos en el taller. En la Encuesta de Alimentación se midieron algunos hábitos de los padres de familia como el consumo de alimentos y la realización de ejercicio. En el Cuestionario para Padres de Familia se les solicitó que emitieran una respuesta dicotómica sobre si sus hijos habían adquirido o no las capacidades listadas después de haber asistido a los talleres.

En una primera sesión, se les explicó a los padres de familia y a los docentes el propósito del Programa ISSSTE en tu Escuela, los beneficios que obtendrían si participaban y se les entregó la Carta de Consentimiento para su firma. Con base en el calendario de la Secretaría de Educación Pública, se elaboró un cronograma para que el equipo multidisciplinario conformado por especialistas médicos, enfermeras, trabajadoras sociales, nutriólogas, psicólogas y activadores físicos asistieran a las ocho EBDI de la Delegación Poniente, una EBDI por mes.

En la primera semana se capacitó a las niñas y niños, en la segunda a los padres de familia y en la tercera, al capital humano de la EBDI. Se impartió un eje temático por día y el orden en que se realizaron los talleres fue: 1) Resiliencia, 2) Nutrición saludable desde la infancia, 3) Salud mental y emocional, 4) Conociendo y cuidado mi cuerpo y 5) Habilidades sociales. El propósito de cada eje, así como algunos de los temas que se revisaron en cada uno de ellos, se presentan en la Tabla 1. Se utilizó material audiovisual y de apoyo elaborado ex profeso para cada uno de los talleres lúdicos-vivenciales. En el caso de las niñas y niños, las encuestas se aplicaron al final de cada taller, mientras que en el caso de los padres de familia se hizo al finalizar la semana.

Se utilizó estadística descriptiva y se reportan porcentajes obtenidos en los ítems enlistados tanto en el Cuestionario para Padres de Familia como en el de la Encuesta de Alimentación. Asimismo, se describió el grado de satisfacción y la elección en cada problema de la Encuesta para Niñas y Niños aplicada en cada taller.

\section{Resultados}

En la tabla 2 se observa el número y el porcentaje de niñas y niños por cada EBDI con respecto al grado de satisfacción y a la elección correcta en cada uno de los ejes temáticos. Al promediar el grado de 
Tabla 2. Niñas y niños capacitados en cada eje temático en las Estancias de Bienestar y Desarrollo Infantil (EBDI)

\begin{tabular}{|c|c|c|c|c|c|c|c|c|c|c|c|c|c|c|c|}
\hline \multirow[t]{3}{*}{ EBDI } & \multicolumn{15}{|c|}{ Ejes } \\
\hline & \multicolumn{3}{|c|}{$\begin{array}{c}I \\
(n=205)\end{array}$} & \multicolumn{3}{|c|}{$\begin{array}{c}\text { II } \\
(n=239)\end{array}$} & \multicolumn{3}{|c|}{$\begin{array}{c}\text { III } \\
(n=232)\end{array}$} & \multicolumn{3}{|c|}{$\begin{array}{c}\text { IV } \\
(n=233)\end{array}$} & \multicolumn{3}{|c|}{$\begin{array}{c}V \\
(n=231)\end{array}$} \\
\hline & Total & $\begin{array}{c}\text { Agrado } \\
\mathbf{n}(\%)\end{array}$ & $\begin{array}{c}\text { Decisión } \\
\mathrm{n}(\%)\end{array}$ & Total & $\begin{array}{c}\text { Agrado } \\
\text { n (\%) }\end{array}$ & $\begin{array}{c}\text { Decisión } \\
\mathrm{n}(\%)\end{array}$ & Total & $\begin{array}{c}\text { Agrado } \\
\mathrm{n}(\%)\end{array}$ & $\begin{array}{c}\text { Decisión } \\
\mathrm{n}(\%)\end{array}$ & Total & $\begin{array}{c}\text { Agrado } \\
\text { n (\%) }\end{array}$ & $\begin{array}{c}\text { Decisión } \\
\mathrm{n}(\%)\end{array}$ & Total & $\begin{array}{c}\text { Agrado } \\
\text { n (\%) }\end{array}$ & $\begin{array}{c}\text { Decisión } \\
\mathrm{n}(\%)\end{array}$ \\
\hline 16 & 35 & $\begin{array}{c}34 \\
(97.14)\end{array}$ & $\begin{array}{c}34 \\
(97.14)\end{array}$ & 31 & $\begin{array}{c}30 \\
(96.77)\end{array}$ & $\begin{array}{c}30 \\
(96.77)\end{array}$ & 30 & $\begin{array}{c}29 \\
(96.67)\end{array}$ & $\begin{array}{c}29 \\
(96.67)\end{array}$ & 32 & $\begin{array}{c}31 \\
(96.88)\end{array}$ & $\begin{array}{c}31 \\
(96.88)\end{array}$ & 28 & $\begin{array}{c}27 \\
(96.43)\end{array}$ & $\begin{array}{c}27 \\
(96.43)\end{array}$ \\
\hline 27 & 16 & $\begin{array}{c}11 \\
(68.75)\end{array}$ & $\begin{array}{c}16 \\
(100)\end{array}$ & 16 & $\begin{array}{c}16 \\
(100)\end{array}$ & $\begin{array}{c}14 \\
(87.50)\end{array}$ & 15 & $\begin{array}{c}14 \\
(93.33)\end{array}$ & $\begin{array}{c}14 \\
(93.33)\end{array}$ & 16 & $\begin{array}{c}15 \\
(93.75)\end{array}$ & $\begin{array}{c}15 \\
(93.75)\end{array}$ & 18 & $\begin{array}{c}17 \\
(94.44)\end{array}$ & $\begin{array}{c}17 \\
(94.44)\end{array}$ \\
\hline 41 & 21 & $\begin{array}{c}21 \\
(100)\end{array}$ & $\begin{array}{c}18 \\
(85.71)\end{array}$ & 40 & $\begin{array}{c}38 \\
(95)\end{array}$ & $\begin{array}{l}38 \\
(95)\end{array}$ & 44 & $\begin{array}{c}40 \\
(90.91)\end{array}$ & $\begin{array}{c}40 \\
(90.91)\end{array}$ & 49 & $\begin{array}{c}49 \\
(100)\end{array}$ & $\begin{array}{c}47 \\
(95.92)\end{array}$ & 40 & $\begin{array}{c}40 \\
(100)\end{array}$ & $\begin{array}{c}39 \\
(97.50)\end{array}$ \\
\hline 46 & 17 & $\begin{array}{c}16 \\
(94.12)\end{array}$ & $\begin{array}{c}17 \\
(100)\end{array}$ & 19 & $\begin{array}{c}19 \\
(100)\end{array}$ & $\begin{array}{c}19 \\
(100)\end{array}$ & 20 & $\begin{array}{c}18 \\
(90)\end{array}$ & $\begin{array}{l}18 \\
(90)\end{array}$ & 13 & $\begin{array}{c}12 \\
(92.31)\end{array}$ & $\begin{array}{c}13 \\
(100)\end{array}$ & 20 & $\begin{array}{c}20 \\
(100)\end{array}$ & $\begin{array}{l}19 \\
(95)\end{array}$ \\
\hline 55 & 36 & $\begin{array}{c}34 \\
(94.44)\end{array}$ & $\begin{array}{c}34 \\
(94.44)\end{array}$ & 38 & $\begin{array}{c}37 \\
(97.37)\end{array}$ & $\begin{array}{c}36 \\
(94.74)\end{array}$ & 40 & $\begin{array}{c}39 \\
(97.50)\end{array}$ & $\begin{array}{c}39 \\
(97.50)\end{array}$ & 37 & $\begin{array}{c}37 \\
(100)\end{array}$ & $\begin{array}{c}37 \\
(100)\end{array}$ & 38 & $\begin{array}{c}36 \\
(94.74)\end{array}$ & $\begin{array}{c}36 \\
(94.74)\end{array}$ \\
\hline 135 & 30 & $\begin{array}{l}27 \\
(90)\end{array}$ & $\begin{array}{c}30 \\
(100)\end{array}$ & 35 & $\begin{array}{c}32 \\
(91.43)\end{array}$ & $\begin{array}{c}33 \\
(94.29)\end{array}$ & 28 & $\begin{array}{c}27 \\
(96.43)\end{array}$ & $\begin{array}{c}27 \\
(96.43)\end{array}$ & 29 & $\begin{array}{c}28 \\
(96.55)\end{array}$ & $\begin{array}{c}28 \\
(96.55)\end{array}$ & 34 & $\begin{array}{c}33 \\
(97.06)\end{array}$ & $\begin{array}{c}32 \\
(94.12)\end{array}$ \\
\hline 155 & 27 & $\begin{array}{c}25 \\
(92.59)\end{array}$ & $\begin{array}{c}26 \\
(96.30)\end{array}$ & 30 & $\begin{array}{c}30 \\
(100)\end{array}$ & $\begin{array}{c}29 \\
(96.67)\end{array}$ & 30 & $\begin{array}{c}28 \\
(93.33)\end{array}$ & $\begin{array}{l}27 \\
(90)\end{array}$ & 29 & $\begin{array}{c}26 \\
(89.66)\end{array}$ & $\begin{array}{c}27 \\
(93.10)\end{array}$ & 27 & $\begin{array}{c}27 \\
(100)\end{array}$ & $\begin{array}{c}27 \\
(100)\end{array}$ \\
\hline 156 & 23 & $\begin{array}{c}23 \\
(100)\end{array}$ & $\begin{array}{c}22 \\
(95.65)\end{array}$ & 30 & $\begin{array}{c}30 \\
(100)\end{array}$ & $\begin{array}{c}29 \\
(96.67)\end{array}$ & 25 & $\begin{array}{c}24 \\
(96)\end{array}$ & $\begin{array}{l}24 \\
(96)\end{array}$ & 28 & $\begin{array}{c}28 \\
(100)\end{array}$ & $\begin{array}{c}28 \\
(100)\end{array}$ & 26 & $\begin{array}{c}26 \\
(100)\end{array}$ & $\begin{array}{c}26 \\
(100)\end{array}$ \\
\hline
\end{tabular}

Ejes: I (Resiliencia), II (Nutrición saludable desde la infancia), III (Salud mental y emocional), IV (Conociendo y cuidando mi cuerpo) y V (Habilidades sociales). El total se refiere al número de niñas y niños capacitados en cada EBDI; la columna Agrado si reportaron que les gustó el taller y en la columna Decisión, si eligieron correctamente la conducta más saludable al final de cada taller.

satisfacción obtenido en los ejes se observó que al 95.59\% (DE: 2.40) de las niñas y niños les agradó el taller; asimismo, que el 95.75\% (DE: 1.25) eligió la opción correcta al problema planteado al final de cada encuesta.

En la Encuesta de Alimentación aplicada a padres de familia (Tabla 3) se observó que, del total de la muestra, el $96.81 \%$ (DE: 0.70) consume verduras, el $95.62 \%$ (DE: 2.77) ingiere frutas, el $94.67 \%$ (DE: 2.82) come carne y el $79.88 \%$ (DE: 3.22) incluye cereales en su dieta. Con respecto a las bebidas gaseosas, se observó que el $41.63 \%$ (DE: 4.36) la incluye en su dieta y que el consumo promedio es de 1.44 vasos (DE: 0.23). Asimismo, se observó que únicamente el $36.21 \%$ (DE: 14.18) realiza algún tipo de ejercicio y que el $63.04 \%$ (DE: 20.27) generó un cambio de hábitos después del taller.

En el Cuestionario para Padres de Familia (Tabla 4) se encontró que los padres reportaron que sus hijos sí desarrollaron las siguientes habilidades y hábitos: resiliencia (media \pm desviación estándar: $84.51 \pm$ $8.88 \%)$, empatía (92.29 $\pm 4.22 \%)$, comunicación asertiva $(93.54 \pm 5.54 \%)$, relaciones interpersonales $(91.07 \pm 4.58 \%)$, toma de decisiones $(92.56 \pm 5.83 \%)$, solución de problemas $(81.05 \pm 12.60 \%)$, pensamiento creativo $(98.06 \pm 2.59 \%)$, pensamiento crítico (86.09 $\pm 9.34 \%$ ), manejo de emociones (80.76 $\pm 11.47 \%$ ), actividad física (97.95 $\pm 2.41 \%$ ), autoconocimiento $(98.96 \pm 2.22 \%)$, hábitos higiénicos $(94.90 \pm 5.34 \%)$ y corresponsabilidad $(86.25 \pm 9.84 \%)$.

\section{Discusión}

El entusiasmo causado por los aspectos positivos que el equipo multidisciplinario observó en esta experiencia y el convencimiento de los propios familiares y docentes que participaron en el desarrollo de los talleres es alentador; aunque la experiencia fue breve y la muestra pequeña, los resultados nos permiten vislumbrar las potencialidades de la metodología. En la evaluación realizada a las niñas y niños, se obtuvo un $95.59 \%$ de satisfacción con respecto a los talleres y un $95.79 \%$ de respuestas correctas en los problemas escritos al final de cada encuesta; en conjunto, estos resultados nos permiten suponer que la sistematización y continua aplicación de esta experiencia permitirá que los asistentes tengan más herramientas para decidir sobre su salud y mejoren sus habilidades para la vida. 
Tabla 3. Hábitos de los familiares después de la capacitación otorgada en el eje «alimentación saludable desde la infancia» en cada una de las Estancias de Bienestar y Desarrollo Infantil (EBDI)

\begin{tabular}{|c|c|c|c|c|c|c|c|c|}
\hline \multirow[t]{2}{*}{ EBDI } & \multirow{2}{*}{$\begin{array}{c}\text { Realiza } \\
\text { ejercicio }(\%)\end{array}$} & \multirow{2}{*}{$\begin{array}{l}\text { Cambió de } \\
\text { hábitos (\%) }\end{array}$} & \multicolumn{5}{|c|}{ Consume (\%) } & \multirow{2}{*}{$\begin{array}{l}\text { Consumo diario de } \\
\text { refresco (promedio) }\end{array}$} \\
\hline & & & Verduras & Frutas & Carne & Cereales & Refresco & \\
\hline 16 & 52.74 & 65.07 & 95.89 & 96.58 & 90.41 & 80.82 & 45.89 & 1.7 \\
\hline 27 & 15.84 & 34.65 & 97.03 & 97.03 & 92.08 & 86.14 & 39.60 & 1.5 \\
\hline 41 & 42.59 & 62.22 & 95.93 & 98.52 & 94.07 & 80.37 & 42.22 & 1.5 \\
\hline 46 & 14.29 & 30.52 & 97.40 & 95.45 & 98.05 & 78.57 & 42.21 & 1.6 \\
\hline 55 & 35.96 & 71.91 & 97.75 & 95.51 & 94.38 & 78.65 & 33.71 & 1 \\
\hline 135 & 46.88 & 77.34 & 96.88 & 95.31 & 96.88 & 78.13 & 46.09 & 1.6 \\
\hline 155 & 36.00 & 86.67 & 97.33 & 89.33 & 93.33 & 81.33 & 45.33 & 1.2 \\
\hline 156 & 45.37 & 75.93 & 96.30 & 97.22 & 98.15 & 75.00 & 37.96 & 1.4 \\
\hline
\end{tabular}

Tabla 4. Reporte de padres de familia acerca de las competencias adquiridas por sus hijas e hijos al terminar el ciclo escolar 2016-2017

\begin{tabular}{|c|c|c|c|c|c|c|c|c|}
\hline \multirow[t]{2}{*}{ Competencias } & \multicolumn{8}{|c|}{ EBDI } \\
\hline & $16(\%)$ & $27(\%)$ & $41(\%)$ & $46(\%)$ & $55(\%)$ & $135(\%)$ & $155(\%)$ & $156(\%)$ \\
\hline Resiliencia & 97.22 & 87.50 & 92.86 & 67.35 & 84.09 & 81.25 & 82.14 & 83.67 \\
\hline Empatía & 97.22 & 93.75 & 90.48 & 95.92 & 95.45 & 84.38 & 89.29 & 91.84 \\
\hline Comunicación asertiva & 97.22 & 100 & 95.24 & 87.76 & 97.73 & 96.88 & 85.71 & 87.76 \\
\hline Relaciones interpersonales & 97.22 & 87.50 & 90.48 & 85.71 & 93.18 & 96.88 & 85.71 & 91.84 \\
\hline Toma de decisiones & 94.44 & 81.25 & 92.86 & 91.84 & 95.45 & 96.88 & 100 & 87.76 \\
\hline Solución de problemas & 91.67 & 81.25 & 83.33 & 53.06 & 86.36 & 84.38 & 92.86 & 75.51 \\
\hline Pensamiento creativo & 100 & 100 & 100 & 97.96 & 97.73 & 100 & 92.86 & 95.92 \\
\hline Pensamiento crítico & 91.67 & 81.25 & 83.33 & 67.35 & 95.45 & 87.50 & 96.43 & 85.71 \\
\hline Manejo de emociones & 88.89 & 93.75 & 78.57 & 61.22 & 79.55 & 87.50 & 89.29 & 67.35 \\
\hline Activación física & 94.44 & 100 & 95.24 & 97.96 & 100 & 100 & 100 & 95.92 \\
\hline Autoconocimiento & 100 & 93.75 & 100 & 100 & 100 & 100 & 100 & 97.96 \\
\hline Hábitos higiénicos & 97.22 & 93.75 & 100 & 83.67 & 100 & 93.75 & 92.86 & 97.96 \\
\hline Corresponsabilidad & 91.67 & 81.25 & 95.24 & 67.35 & 95.45 & 93.75 & 85.71 & 79.59 \\
\hline
\end{tabular}

EBDI: Estancias de Bienestar y Desarrollo Infantil.

Este estudio representa una primera aproximación al fortalecimiento del binomio salud-educación y abarca tanto la esfera individual como la familiar y escolar. La familia es la estructura de la sociedad responsable de la salud de sus integrantes y en ella se generan los procedimientos para su conservación, fomento y recuperación ${ }^{27}$, por lo tanto, su fortalecimiento se vuelve una prioridad de los sistemas de salud.

La intervención en preescolares se decidió porque es en la infancia cuando se aprenden las normas y los valores; además, es el periodo en el que se imitan los modelos (familia o docentes), se ensayan los roles, se forma el autoconcepto y se aprende el manejo de emociones, las conductas prosociales y las habilidades de comunicación y negociación ${ }^{28-30}$. Así, enseñar y fortalecer algunos de los pilares de la resiliencia (autoestima, introspección, independencia, capacidad de relacionarse y de pensamiento crítico, iniciativa, humor, creatividad, moralidad) y de las habilidades para la vida (autoconocimiento, empatía, comunicación asertiva, relaciones interpersonales, toma de decisiones, pensamiento creativo y crítico, manejo de problemas, conflictos, emociones, sentimientos y estrés) ${ }^{31}$ permitió mejorar el clima de convivencia familiar y escolar. La autoconfianza, la autoestima, la seguridad, la capacidad de compartir 
y amar, e incluso las habilidades intelectuales y sociales, tienen sus raíces en las experiencias vividas durante la primera infancia en el seno familiar y escolar. Una sociedad violenta, la disfunción familiar, estilos de crianza negligentes o autoritarios, o bien reglas poco claras en el hogar, falta de comunicación o poca supervisión y acompañamiento de los hijos pueden generar problemas psicológicos, adicciones, violencia o desensibilización a la violencia ${ }^{32-35}$. En el estudio se observó una corresponsabilidad de los docentes y de los padres de familia en la salud física y mental de las niñas y niños preescolares debido no solo a su asistencia al Programa, sino a su participación activa, ya que el $63.04 \%$ reportó haber generado un cambio en sus hábitos después de asistir a los talleres. En los lugares donde se respira un ambiente de cariño, respeto, confianza y estabilidad, los niños y niñas se crían y se desarrollan psíquicamente más sanos y seguros, se relacionarán con el exterior de esta misma forma y con una actitud más positiva y constructiva hacia la vida ${ }^{36}$.

Este estudio se inserta en el concepto de promoción de la salud, que es el proceso que permite a las personas incrementar el control sobre su salud para mejorarla, y abarca tanto las acciones dirigidas a incrementar las habilidades y capacidades de las personas como las destinadas a modificar las condiciones sociales, ambientales y económicas para que esta mejore. La educación para la salud es una parte del proceso asistencial que incluye la prevención, el tratamiento y la rehabilitación; por lo tanto, comprende las oportunidades de aprendizaje destinadas a mejorar la alfabetización sanitaria al incluir una población informada con habilidades personales que conduzcan a la toma consciente de decisiones que mejoren su calidad de vida. Educar en salud es un proceso que tiene como finalidad generar ciudadanos que se responsabilicen en la defensa de la salud propia y colectiva y, por lo tanto, es parte de las funciones de los profesionales sanitarios, sociales y de la educación ${ }^{37}$. Aunque este estudio representa una primera aproximación al binomio salud-educación en las EBDI, se puede observar que en futuras intervenciones se tendrán que fortalecer los programas para generar mayor conciencia en los asistentes y se modifiquen algunos de los hábitos. Por ejemplo, solo el $36.21 \%$ de la muestra practica algún tipo de ejercicio y el $41.63 \%$ consume alguna bebida gaseosa; ambas conductas favorecen la aparición del sobrepeso y la obesidad, lo cual se considera un factor de riesgo para la aparición de enfermedades crónicas no transmisibles.
EI Programa ISSSTE en tu Escuela coincide con las propuestas de la Red de Escuelas para la Salud en Europa fundamentada en la experiencia desarrollada por su precursora, la Red Europea de Escuelas Promotoras de Salud. Esto ha facilitado el trabajo estratégico entre los sectores educativo y sanitario lo que ha elevado el perfil y el compromiso con la promoción de salud en la escuela ${ }^{40}$. El Programa reconoce la Carta de las Naciones Unidas de los Derechos de la Infancia ${ }^{42}$ y promueve acciones para elevar la calidad de la educación y de la salud. La revisión de la literatura ${ }^{43-46}$ y de diferentes modelos ecológicos ${ }^{46}$ apoya la eficacia del enfoque de las escuelas para la salud, así como su impacto a nivel social.

\section{Conclusión}

El Programa ISSSTE en tu Escuela muestra que existe un potencial incalculable para el desarrollo de niñas, niños, padres de familia y docentes resilientes, participativos y solidarios para que se empoderen de su salud. Esta experiencia pretende ser una contribución a ese desarrollo, y en tanto tal, consideramos que puede ser exitosa. Este programa es una contribución al camino de colaboración entre los distintos sectores responsables para que nuestra sociedad tenga una vida de mayor calidad en salud. Se considera la conveniencia de la aplicación transexenal de este tipo de trabajos y que se realice en todos los niveles educativos, ya que esto garantizaría un impacto mayor en sus resultados a corto, mediano y largo plazo.

\section{Agradecimientos}

Por su colaboración y apoyo en el desarrollo del trabajo al equipo multidisciplinario ISSSTE en tu escuela y al personal de las Estancias de Bienestar y Desarrollo Infantil de la Delegación Regional Poniente del ISSSTE.

\section{Bibliografía}

1. FUNDADEPS. La familia como agente de salud. 1. a Ed. Madrid: $I O$ Sistemas de Comunicación; 2012.

2. Munist $M$, Santos $H$, Kotliarenco $M$, Suárez Ojeda $E$, Infante $F$, Grotberg E. Manual de identificación y promoción de la resiliencia en niños y adolescentes. Washington, D.C.: Fundación WK Kellog; 1998.

3. Munist M, Suárez Ojeda N, Krauskopf D, Silber T. Adolescencia y resilencia. México: Paidos, 2007.

4. Better policies for better lives [Internet]. Organisation for Economic Co-operation and Development; 2011. Disponible en: https://wwwoecdorg/about/47747755pdf

5. Barrera-Cruz A, Rodríguez-González A, Molina-Ayala MA. Escenario actual de la obesidad en México. Rev Med Inst Mex Seguro Soc. 2013;51:292-9. 
6. Barriguete-Meléndez JA, Unikel-Santoncini C, Aguilar-Salinas C, Córdoba-Villalobos JA, Shamah T, Barquera S, et al. Prevalence of abnormal eating behaviors in adolescents in Mexico: Mexican National Health and Nutrition Survey 2006. Salud Publica Mex. 2009;51(Suppl 4):S638-44.

7. INEGI. Encuesta Nacional de la Dinámica Demográfica 2014 [Internet]. INEGI; 2014. Disponible en: http://wwwinegiorgmx/saladeprensa/boletines/2015/especiales/especiales2015_07_1pdf

8. Cancino AMM, Valencia MH. Embarazo en la adolescencia: cómo ocurre en la sociedad actual. Perinatol Reprod Hum. 2015;29:76-82.

9. Orientaciones para la prevención, detección y actuación en casos de abuso sexual infantil, acoso escolar y maltrato en las escuelas de Educación Básica [Internet]. México: Secretaría de Educación Pública; fecha documento [fecha de consulta o de última actualización]. Disponible en: www.gob.mx/cms/uploads/docs/Orientaciones_211216.pdf

10. Fatima Kantun Puc L, Peña Castillo RF. Prevención del abuso sexual en una comunidad maya de Yucatán, México: relevancia de las características socioculturales (2014). Rev Crim. 2015;57:74-90.

11. Albores-Gallo $\mathrm{L}$. Autolesiones sin intención suicida en una muestra de niños $y$ adolescentes de la ciudad de México. Actas Esp Psiquiatr. 2014;42(4):159-68.

12. Encuesta Nacional de Consumo de Drogas, Alcohol y Tabaco 20162017. México: Pública INdS. Disponible en: wwwinspmx/avisos/4585-encodat-2016html.

13. Sánchez-Hernández, Ó., Méndez, F., \& Garber, J. (2014). Prevención de la depresión en niños y adolescentes: Revisión y reflexión. Revista de Psicopatología y Psicología Clínica, 19(1), 63-76.

14. Garibay-Ramirez J, Jiménez-Garcés $C$, Vieyra-Reyes $P$, Hernández-González MM, Villalón-López J. Disfunción familiar y depresión en niños de 8-12 años de edad. Rev med investig Univ Autón Estado Méx. 2014:2:107-11.

15. Acosta-Hernández M, Mancilla-Percino T, Correa-Basurto J, Saavedra-Vélez M, Ramos-Morales F, Cruz-Sánchez JS, et al. Depresión en la infancia y adolescencia: enfermedad de nuestro tiempo. Archivos de neurociencias, 16(3), 156-161.

16. Principales causas de mortalidad por residencia habitual, grupos de edad y sexo del fallecido [Internet]. México: INEGI; 2015. Disponible en: http:// wwwinegiorgmx/est/contenidos/proyectos/registros/vitales/mortalidad/tabulados/ConsultaMortalidadasp.

17. Villatoro Velázquez J, Bustos Gamiño M, Oliva Robles N, Fregoso Ito D, Mujica Salazar A, Martín del Campo Sánchez R, et al. Encuesta Nacional de Consumo de Drogas en Estudiantes 2014: Reporte de Tabaco [Internet]. México: Instituto Nacional de Psiquiatría Ramón de la Fuente Muñiz México; 2015. Disponible en: https://wwwgobmx/cms/uploads/attachment/file/239258/ENCODE TABACO 2014pdf.

18. Villatoro Velázquez J, Bustos Gamiño M, Oliva Robles N, Fregoso Ito D, Mujica Salazar A, Martín del Campo Sánchez R, et al. Encuesta Nacional de Consumo de Drogas en Estudiantes 2014: Reporte de Alcoho [Internet]. México: Instituto Nacional de Psiquiatría Ramón de la Fuente Muñiz México; 2014. Disponible en: https://wwwgobmx/cms/uploads/attachment/file/239257/ENCODE ALCOHOL 2014pdf

19. Villatoro-Velázquez J, Bustos Gamiño M, Oliva Robles N, Fregoso Ito D, Mujica Salazar A, Martín del Campo Sánchez R, et al. Encuesta Nacional de Consumo de Drogas en Estudiantes 2014: Reporte de Drogas [Internet]. México: Instituto Nacional de Psiquiatría Ramón de la Fuente Muñiz México; 2014. Disponible en: http://wwwconadicsaludgobmx/pdfs/ investigacion/ENCODE_DROGAS_2014pdf.

20. Santoyo Castillo D, Frías SM. Acoso escolar en México: actores involucrados y sus características. 2014.

21. Márquez-Caraveo ME, Arroyo-García E, Granados-Rojas A, Ángeles-Llerenas AJ. Hospital Psiquiátrico Infantil Dr. Juan N. Navarro: 50 años de atención a la salud mental de niños y adolescentes en México. 2017; 59:477-84.

22. Sauceda García JM. Trastorno por déficit de atención con hiperactividad: un problema de salud pública. 2014;57:14-9.

23. Grotberg E. A guide to promoting resilience in children: strengthening the human spirit [Internet]. La Haya, Países Bajos: Bernard Van Leer
Foundation; 1995. Disponible en: https://bibalexorg/baifa/Attachment/ Documents/115519pdf

24. Gil GE. La resiliencia: conceptos y modelos aplicables al entorno escolar. 2010;19:27-42.

25. Vilar J, Pont EJ. Reflexiones en torno a la resiliencia. Una conversación con Stefan Vanistendael. 2009:93-103.

26. Windle GJ. What is resilience? A review and concept analysis. 2011; 21:152-69.

27. Louro Bernal IJ. La familia en la determinación de la salud. 2003;29:48-51.

28. Ternera LA. El desarrollo del autoconcepto en niños y niñas y su relación con la interacción social en la infancia. 2014;17:67-9.

29. Isaza-Valencia L, Henao-López CG. Influencia del clima sociofamiliar y estilos de interacción parental sobre el desarrollo de habilidades sociales en niños y niñas. 2012:253-71.

30. Cuervo Martinez Á. Pautas de crianza y desarrollo socioafectivo en la infancia. 2010;6.

31. Mangrulkar L, Whitman CV, Posner M. Enfoque de habilidades para la vida para un desarrollo saludable de niños y adolescentes Washington, DC: OPS; 2001.

32. Fuentes MC, Alarcón A, García F, Gracia EJ. Consumo de alcohol, tabaco, cannabis y otras drogas en la adolescencia: efectos de la familia y peligro del barrio. 2015;31:1000-7.

33. Ramírez Ruiz M, de Andrade DJ. La familia y los factores de riesgo relacionados con el consumo de alcohol y tabaco en los niños y adolescentes (Guayaquil-Ecuador). 2005;13.

34. Gallegos WL. Agresión y violencia en la adolescencia: La importancia de la familia. 2013:21:23-34

35. Jiménez JSFG, Serrano MdLPJU. Desensibilización a la violencia una revisión teórica para la delimitación de un constructo. 2017;11:70-81.

36. UNICEF. Desarrollo psicosocial de los niños y las niñas. 2. ${ }^{a}$ Ed [Internet]. UNICEF, Oficina de Área para Colombia y Venezuela; 2004. Disponible en: https://wwwuniceforg/colombia/pdf/ManualDPpdf

37. Promoción de la Salud: Glosario [Internet]. México: Ministerio de Sanidad y Consumo; 1998. Disponible en: https://wwwmsssigobes/profesionales/ saludPublica/prevPromocion/docs/glosariopdf

38. NIGZ, Centro Colaborador de la OMS para la Promoción de la Salud Escolar. Red de Escuelas para la Salud en Europa, red SHE. Coordina-ción en España. 2017.

39. Derechos Humanos. Convención sobre los Derechos de los Niños [lnternet]. Organización de las Naciones Unidas; fecha. Disponible en: http://www.ohchr.org/SP/Professionallnterest/Pages/CRC.aspx

40. Jourdan D, Mannix McNamara P, Simar C, Pommier J. Factors influencing the contribution of staff to health education in schools. Health Educ Res. 2010;25(4):519-30.

41. Fondo de las Naciones Unidas para la Infancia UNICEF. Convención sobre los derechos de los niños. 4a Ed. México. 2002. Disponible: http:// sitios.dif.gob.mx/difusoresnacionales/wp-content/uploads/2015/10/ConvencionDerechosNinos.pdf

42. Patino-Fernández AM, Hernández J, Villa M, Delamater A. School-based health promotion intervention: parent and school staff perspectives. J Sch Health. 2013;83:763-70.

43. Ramos P, Pasarín MI, Artazcoz L, et al. Escuelas saludables y participativas: evaluación de una estrategia de salud pública. Gac Sanit. 2013; 27:104-10.

44. Jourdan D, Pironom J, Berger D, et al. Factors influencing teachers' views of health and health education: a study in 15 countries. Health Educ J. 2013;72:660-72.

45. Bronfenbrenner U, Morris PA. The bioecological model of human development. En: Lerner RM, editor. Handbook of child psychology. Vol. 1. Theoretical models of human development. Hoboken, NJ: John Wiley \& Sons; 2006. pp. 793-828.

46. Pérez GA. Paradigmas contemporáneos de investigación didáctica, en Gimeno SJ y Perez GA. La enseñanza: su teoría y su práctica, Madrid: Akal editor. 1985 pp. $125-138$. 